REVIEW

\title{
Postoperative ileus: strategies for reduction
}

\author{
James Lubawski \\ Theodore Saclarides \\ Section of Colon and Rectal Surgery, \\ Rush University Medical Center, \\ Chicago, IL, USA
}

\begin{abstract}
Postoperative Ileus (POI) is a frequent, frustrating occurrence for patients and surgeons after abdominal surgery. Despite significant research investigating how to reduce this multi-factorial phenomenon, a single strategy has not been shown to reduce POI's significant effects on length of stay (LOS) and hospital costs. Perhaps the most significant cause of POI is the use of narcotics for analgesia. Strategies that target inflammation and pain reduction such as NSAID use, epidural analgesia, and laparoscopic techniques will reduce POI but are accompanied by a simultaneous reduction in opioid use. Pharmacologic means of stimulating gut motility have not shown a positive effect, and the routine use of nasogastric tubes only increases morbidity. Recent multi-site phase III trials with alvimopan, a peripherally acting mu-antagonist, have shown significant reductions in POI and LOS by 12 and 16 hours, respectively, by blunting the effects of narcotics on gut motility while sparing centrally mediated analgesia. Use of alvimopan, along with a multi-modal postoperative treatment plan involving early ambulation, feeding, and avoiding nasogastric tubes, will likely be the crux of POI treatment and prevention.
\end{abstract}

Keywords: postoperative ileus, alvimopan, abdominal surgery

\section{Introduction}

Despite numerous advances in surgical technique and perioperative care, postoperative ileus (POI) continues to be one of the most common and expected aspects of abdominal surgery. It prolongs hospital stays, increases medical costs and frustrates patients and surgeons (Schuster et al 2006; Viscusi et al 2006). Many authors suggest that POI is a mandatory phase of the recovery period for any intra-abdominal procedure, and only an ileus lasting greater than 5 days is abnormal - to be termed a prolonged POI (Holke and Kehlet 2000; Delaney 2004; Sajja and Schein 2004). Under this definition, fully $40 \%$ of patients undergoing laparotomy experience prolonged POI (Delaney 2004). Whether or not it is accepted that POI is a mandatory aspect of surgery, surgeons are continually trying to find ways to shorten this period. Traditional methods of providing postoperative care have included bowel rest and nasogastric (NG) tube decompression. These methods, despite their continued prevalence, have been shown to be ineffective and unnecessary and, if anything, increase morbidity (Cheatham et al 1995; Braga et al 2002; Nelson et al 2005). Methods for shortening POI target different aspects of its multi-factorial etiology.

Prior to 1990, very few articles were published on length of stay issues. None were clinical trials or discussed methods to shorten hospital stay. Since 1995, however, a plethora of articles has been published which have included randomized, prospective studies, and cohort comparison trials. Hospital stay is determined by the need for pain control, the presence of nausea and vomiting, fatigue, mechanical factors such as surgical drains and stomas, organ dysfunction, and the speed with which diet can be advanced. The presence of a postoperative ileus is a major determinant of the length of hospital stay. All of these issues have been addressed and methods devised to reduce their impact. With nearly 350,000 colorectal and small bowel resections occurring 
annually, at an average stay of almost 11 days at a bill to the healthcare system of greater than US \$20 billion, the cost savings could be substantial if length of stay was reduced by only 1 to 2 days in each case (Healthcare Costs 2005).

In its simplest form, ileus is defined as the inhibition of propulsive bowel activity and is manifested by abdominal distention, nausea, vomiting, and diet intolerance. Of the divisions of the gastrointestinal tract, the small intestine resumes its normal peristaltic activity within 24 hours, followed by the stomach (24-48 hours). The colon can take up to 120 hours for normal motility (Miedema and Johnson 2003). The true incidence of postoperative ileus is not known because of incomplete documentation but is highest in procedures involving small bowel and large bowel resections.

The difficulty in attempting to prevent or relieve POI is in part due to an etiology influenced by intrinsic and extrinsic mechanisms. Inflammatory, hormonal, and pharmacologic mechanisms have all been show to play a role in the onset of POI. Currently, multi-modal regimens that target multiple mechanisms have shown the greatest success in reducing POI (Basse et al 2000, 2003; Delaney et al 2001). Those by Basse et al suggest that return to bowel function can be reduced to 2 days in $90 \%$ of patients (Basse et al 2003). However, the success of each component of these multimodal regimens is limited if narcotics are used for the necessary but sometimes detrimental relief of pain. Up until now, there has been no pharmaceutical agent that has been consistently effective in hastening bowel recovery from abdominal surgery. This may change as alvimopan, a peripherally acting mu-opioid antagonist, is proving its worth in reducing the effects of narcotic medications on bowel motility.

The origins of POI are multifactorial. Basic intestinal motility patterns are intrinsically determined by the enteric nervous system. Extrinsic control is via the autonomic nervous system which can either accelerate or retard these motility patterns. Inflammatory mediators released in response to surgical manipulation, and infection can also affect bowel motility. Endogenous and exogenous opioids reduce propulsive activity in the gastrointestinal tract. The former are released as part of the stress response in the postoperative period. Both types of opioids activate the same receptor sites in the gut and affect a variety of functions including peristaltic activity, secretion, transport of electrolytes and fluids, and gastric emptying.

Management strategies for POI can be divided into prevention and supportive care. For prevention, one can alter the choice of anesthesia, the surgical technique, and the means of providing pain relief. For supportive care, considerable research has looked into avoiding the use of routine NG intubation, early ambulation, early oral feeding, and prokinetic agents. These strategies have been incorporated into fast track protocols designed to shorten POI and hasten discharge. For some of these components, evidence is strong for their use, for others it is less so. Again, the limiting factor seems to be the inhibitory affect of narcotics.

\section{Inflammatory}

The act of performing a surgical procedure on the bowel activates a pro-inflammatory cascade of events within intestinal tissues. Latent macrophages become activated with bowel manipulation. This increases production of nitric oxide (NO), inflammatory cytokines and prostaglandins via the cyclo-oxygenase-2 pathway (COX-2), all of which lead to leukocyte recruitment to the bowel wall. The end effect is suppression of bowel muscle function (Bauer and Boeckxstaens 2004).

\section{NSAIDS}

Changes in the pharmacologic management of patients undergoing abdominal surgery have been tried to reduce the inflammatory component of the procedure and thus POI. With the large role the COX-2 pathway plays in the inflammatory process, it has been hypothesized that use of COX-2 inhibitors would help to curb depression of bowel mobility. Results of these studies have been mixed, with there either being no effect or a slight reduction in POI time. Those studies that have shown a benefit in using COX-2 inhibitors have not been able to demonstrate that the reduction in POI is independent of the subsequent reduction in opioid use. The main benefit of these drugs has been their ability to lower the total dose of narcotics needed for adequate pain control (Bouras et al 2004; Sim et al 2007). Similar trials have occurred using the intravenous anti-inflammatory drug, ketorolac. Use of ketorolac does have an opioid sparing effect but does little to reduce the duration of POI (Chen et al 2005).

\section{Laparoscopy}

Numerous studies have shown that laparoscopic surgery reduces the duration of POI (Lacy et al 1995; Milsom et al 1998; Bass et al 2003). The inflammatory pathway described above was found to increase as surgery progressed and early research tried to show that laparoscopic techniques would also reduce inflammation and thus POI by reducing incision size and bowel handling (Kalff et al 1998; Bauer and Boeckxstaens 2004). When examined separately, incision length and bowel handling could not be shown 
to have a measurable effect on POI. Much like the problems involved in anti-inflammatory drug studies, incision length studies could not show that reductions of POI seen in more minimally invasive surgeries were independent of a subsequent decrease in morphine use (Cali et al 1995; Delaney 2004).

\section{Hormonal/metabolic}

Laparotomy and surgical manipulation of the bowel induces a metabolic stress response which stimulates the sympathetic nervous system and adrenergic pathways (Correia and da Silva 2004). With this in mind, laboratory trials have set out to determine if any medications that block these pathways could be effective. Beta-blockers have been shown to have no effect on the duration of POI in animal models (Bauer and Boeckxstaens 2004; Uemura et al 2004). Yohimbine does, however, improve gastrointestinal (GI) transit in rats suggesting that the alpha-2 adrenoreceptor is the key to the sympathetic response (Uemura et al 2004). The anti-acetylcholinesterase effects of neostigmine have also been shown to improve symptoms of POI (Bauer and Boeckxstaens 2004).

\section{Epidural anesthesia}

Another mechanism used to block the influence of sympathetic nerves on the gut is thoracic epidural anesthesia. Infusion of local anesthetics into the epidural space blocks sympathetic stimulation while preserving parasympathetic innervation (Fotiadis et al 2004). The GI motility enhancing parasympathetic system stems from the vagus nerve whose cranial origin is unaffected by epidural anesthesia. This theoretical benefit ends at the splenic flexure after which point the colon is controlled by parasympathetic nerves with sacral roots. Analysis of the Cochrane database has shown a decrease in POI with epidural anesthesia of over 12 hours. However, this did not translate into a reduction in LOS (Jorgensen et al 2000).

\section{Early nutrition}

It has been shown recently that non-pharmacologic mechanisms for reducing the stress response are quite successful. Early enteral feeding in the immediate postoperative period is gaining favor as an increasing number of studies are showing that it is well tolerated in greater than $80 \%$ of patients and decreases both POI and LOS (Choi and O'Donnell 1996; Velez et al 1997; Stewart et al 1998). The theory supporting early feeding is that it stimulates gastrointestinal hormones, elicits gut propulsive activity, and thus coordinated gut motility. Concern over an increased incidence of anastomotic leaks with early feeding is unfounded (Correia and da Silva 2004). In fact, early feeding reduces the risk of infection and may shorten hospital stay. Stimulation of bowel motility without use of food has shown some success with gum chewing. The chewing serves to act a sham feeding. It is postulated that this stimulates the cephalic-vagal reflex translating to an increase in GI hormone production; time to resolution of POI and hospital discharge are both reduced (Asao et al 2002; Schuster et al 2006).

\section{Nasogastric decompression}

Placement of a NG tube for decompression may alleviate symptoms of a POI once they occur. There is no evidence, however, that routine placement of a NG tube at surgery will prevent an ileus or shorten its duration. They may, in fact, increase the incidence of postoperative pulmonary complications (Cheatham et al 1995; Nelson et al 2005).

\section{Pharmacologic}

To date, pharmacologic agents have had little to no effectiveness in minimizing or preventing POI (Bungard and Kale-Pradham 1999). Metoclopramide, a dopamine antagonist and cholinergic agonist, has not been shown to have a beneficial affect in most randomized controlled trials (Set and Kale-Pradham 2001). Side affects include drowsiness, dystonic reactions, and agitation. Cisapride showed modest efficacy; however, it was withdrawn from the US market due to its cardiovascular side affects. Erythromycin, a motilin agonist, has not been beneficial (Smith et al 2000). Antiadrenergic agents block the sympathetic neural reflex but have little practical use in POI because of potential cardiovascular side affects. The same is true for cholinergic agents; however, neostigmine has shown some utility in the treatment of colonic pseudo-obstruction. Laxatives (stimulant, osmotic, bulking) have not been extensively studied in randomized, prospective trials and are not part of most fast-track protocols.

\section{Opioids}

Many of the previously stated methods to reduce POI are confounded by one factor: opioid use. Opioids inhibit acetylcholine release, reduce gastrointestinal motility and directly lengthen POI with increasing dosages (Cali et al 1995; Baig and Wexter 2004; Harms and Heise 2007). Smaller, laparoscopic incisions, increased use of NSAID analgesia and epidural anesthesia, while all theoretically 
useful in reducing POI through various mechanisms, are also effective reducers of opioid use.

\section{Alvimopan}

Alvimopan, a peripherally acting mu-opioid antagonist, has recently shown great promise in phase III studies as a treatment for POI (Delaney et al 2005). The polarity of the alvimopan molecule does not permit it to cross the blood-brain barrier. Thus, it does not interfere with centrally mediated effects of opioids - including analgesia. It does, however, interfere with the peripheral effect of opioids upon GI tract motility (Leslie 2005).

Phase III studies have occurred at a number of centers, and have studied patients greater than 18 years of age who have undergone bowel resection or hysterectomy. Three groups were studied: those receiving placebo, $6 \mathrm{mg}$ of alvimopan or $12 \mathrm{mg} 2$ hours before the procedure and up to one week after. The primary outcome measure was termed GI-3, which was measured at the latter of two events: first toleration of solid food and time to first flatus or bowel movement. All of the phase III trials were conducted in conjunction with a multi-modal, fast-track treatment regimen which included ambulation and fluids on POD \#1, solids on POD \#2, morphine PCA, and no postoperative NG tube (Tan et al 2007).

Meta analysis of alvimopan phase III trials showed a significant improvement in time to first bowel movement, flatus, and toleration of solid food. Overall, there was a greater than 12-hour reduction in time to recover GI function (as defined by GI-3). Furthermore, there was an improvement in hospital discharge by greater than 16 hours. The placebo and study groups showed no difference in overall opioid use or in reported pain scores supporting alvimopan's ability to block the GI but not the analgesic effects of narcotic medications. At higher doses alvimopan use led to a decrease in nausea and vomiting and the need for NG tube insertion. Hospital re-admission was lower with alvimopan compared with placebo (Delaney 2004; Wolff et al 2004; Delaney et al 2007).

This is a promising result for a condition which has resisted all medical management up to this point. It should be noted again that all of the phase III trials were conducted using a multi-modal, fast-track care path. This resulted in a reduced length of stay compared to the average for bowel resection in all the groups, including the placebo group. This correlates well to the results found by CREAD (Controlled Rehabilitation with Early Ambulation and Diet) and other multi-modal studies (Delaney et al 2003). In addition to early diet and ambulation, CREAD gave patients a morphine PCA and ketorolac for pain, no epidural anesthesia or NG tube.

\section{Conclusion}

If approved for clinical use, phase III studies indicate that alvimopan will likely become a useful adjunct to postoperative care of patients undergoing colorectal surgery. Until that time multi-modal, fast-track approaches hold the greatest promise for POI reduction. As laid out in the alvimopan trails, this consists of: no nasogastric tube, morphine PCA and ketorolac for pain control, ambulation and liquids on POD \#1, and solid food on POD \#2. This regimen reduced length of stay by an average of 5 days, with alvimopan further reducing that time by 12 hours or more (Delaney et al 2007).

\section{Disclosures}

The authors report no conflicts of interest.

\section{References}

Asao T, Kuwano H, Nakamura J, et al. 2002. Gum chewing enhances early recovery from postoperative ileus after laparoscopic colectomy. $\mathrm{J} \mathrm{Am}$ Coll Surg, 195:30-2.

Baig MK, Wexner SD. 2004. Postoperative ileus: a review. Dis Colon Rectum, 47:516-26.

Bauer AJ, Boeckxstaens GE. 2004. Mechanisms of postoperative ileus. Neurogastroenterol Motil, 16(Suppl 2):54-60.

Basse L, Madsen JL, Billesbolle P, et al. 2003. Gastrointestinal transit after laparoscopic versus open colonic resection. Surg Endosc, 17:1919-22.

Basse L, Hor Jakobsen D, Billesbolle P, et al. 2000. A clinical pathway to accelerate recovery after colonic resection. Ann Surg, 232:51-7.

Basse L, Thorbol JE, Lossi K, et al. 2004. Colonic surgery with accelerated rehabilitation or conventional care. Dis Colon Rectum, 47:271-7.

Bouras EP, Burton DD, Camilleri M, et al. 2004. Effect of cyclooxygenase-2 inhibitors on gastric emptying and small intestinal transit in humans. Neurogastroenterol Motil, 16:729-35.

Braga M, Gianotti L, Gentilini O, et al. 2002. Feeding the gut early after digestive surgery: results of a nine-year experience. Clin Nutr, 21:59-65.

Bungard TJ, Kale-Pradhan PB. 1999. Prokinetic agents for the treatment of postoperative ileus in adults: a review of the literature. Pharmacotherapy, 19:416-423.

Cali RL, Meade PG, Swanson MS, et al. 1995. Effect of morphine and incision length on bowel function after colectomy. Dis Colon Rectum, 43:163-8.

Cheatham ML, Chapman WC, Key SP, et al. 1995. A meta-analysis of selective versus routine nasogastric decompression after elective laparotomy. Ann Surg, 221:469-76.

Chen JY, Wu GJ, Mok MS, et al. 2005. Effect of adding ketorolac to intravenous morphine patient-controlled analgesia on bowel function in colorectal surgery patients - a prospective, randomized, double-blind study. Acta Anaesthesiol Scand, 49:546-51.

Choi J, O'Connell TX. 1996. Safe and effective early postoperative feeding and hospital discharge after open colon resection. Am Surg, 62:853-6.

Correia MI, da Silva RG. 2004. The impact of early nutrition on metabolic response and postoperative ileus. Curr Opin Clin Nutr Metab Care, 7:577-83. 
Delaney CP, Fazio VW, Senagore AJ, et al. 2001. 'Fast track' postoperative management protocol for patients with high co-morbidity undergoing complex abdominal and pelvic colorectal surgery. $\mathrm{Br} J$ Surg, 88:1533-8.

Delaney CP, Zutshi M, Senagore AJ, et al. 2003. Prospective, randomized, controlled trial between a pathway of Controlled Rehabilitation with Early Ambulation and Diet (CREAD) and traditional postoperative care after laparotomy and intestinal resection. Dis Colon Rectum, 46:851-9.

Delaney CP. 2004. Clinical perspective on postoperative ileus and the effect of opiates. Neurogastroenterol Motility, 16(s2):61-6.

Delaney, CP, Weese JL, Hyman NH, et al. 2005. Phase III trial of alvimopan, a novel, peripherally acting, mu opioid antagonist, for postoperative ileus after major abdominal surgery. Dis Colon Rectum, 48:1114-29.

Delaney CP, Wolff BG, Viscusi ER, et al. 2007. Alvimopan for postoperative ileus following bowel resection: a pooled analysis of phase III studies. Ann Surg, 245:355-63.

Fotiadis RJ, Badvie S, Weston MD, et al. 2004. Epidural analgesia in gastrointestinal surgery. Br J Surg, 91:828-41.

Harms BA, Heise CP. 2007. Pharmacologic Management of Postoperative Ileus; The Next Chapter in Surgery. Ann Surg, 245:364-5.

Healthcare Costs and Utilization Project. 2005. National Statistics [online]. URL: http://hcupnet.ahrq.gov/. Accessed February 3, 2008.

Holte K, Kehlet H. 2000. Postoperative ileus: a preventable event. Br J Surg, $87: 1480-93$

Jorgensen H, Wetterslev J, Moiniche S et al. 2000. Epidural local anaesthetics versus opioid-based analgesic regimens for postoperative gastrointestinal paralysis, PONV and pain after abdominal surgery. Cochrane Database Syst Rev, (4):CD001893.

Kalff JC, Schraut WH, Simmons RL, et al. 1998. Surgical manipulation of the gut elicits an intestinal muscularis inflammatory response resulting in postsurgical ileus. Ann Surg, 228:652-63.

Lacy AM, Garcia-Valdecasas JC, Pique JM, et al. 1995. Short-term outcome analysis of a randomized study comparing laparoscopic vs open colectomy for colon cancer. Surg Endosc, 9:1001-5.

Leslie JB. 2005. Alvimopan for the management of postoperative ileus. Ann Pharmacother, 39:1502-10.

Miedema BW, Johnson JO. 2003. Methods for decreasing postoperative gut dysmotility. Lancet Oncol, 4:365-72.

Milsom JW, Bohm B, Hammerhofer KA, et al. 1998. A prospective, randomized trial comparing laparoscopic versus conventional techniques in colorectal cancer surgery: a preliminary report. $J \mathrm{Am}$ Coll Surg, 187:46-54.
Nelson R, Edwards S, Tse B. 2005. Prophylactic nasogastric decompression after abdominal surgery. Cochrane Database Syst Rev, 25(1):CD004929.

Nelson R, Tse B, Edwards S. 2005. Systematic review of prophylactic nasogastric decompression after abdominal operations. Br J Surg, 92:673-80.

Sajja SB, Schein M. 2004. Early postoperative small bowel obstruction. Br J Surg, 91:683-91.

Schuster R, Grewal N, Greaney GC, et al. 2006. Gum chewing reduces ileus after elective open sigmoid colectomy. Arch Surg, 141:174-6.

Seta ML, Kale-Pradhan PB. 2001. Efficacy of metoclopramide in postoperative ileus after exploratory laparotomy. Pharmacotherapy, 21:1181-6.

Sim R, Cheong DM, Wong KS, et al. 2007. Prospective randomized, doubleblind, placebo-controlled study of pre- and postoperative administration of a COX-2-specific inhibitor as opioid-sparing analgesia in major colorectal surgery. Colorectal Dis, 9:52-60.

Smith AJ, Nissan A, Lanouette NM, et al. 2000. Prokinetic effect of erythromycin after colorectal surgery: randomized, placebo-controlled, double blind study. Dis Colon Rectum, 43:333-7.

Stewart BT, Woods RJ, Collopy BT, et al. 1998. Early feeding after elective open colorectal resections: a prospective randomized trial. Aust N Z J Surg, 68:125-8.

Tan EK, Cornish J, Darzi AW, et al. 2007. Meta-analysis: Alvimopan vs placebo in the treatment of post-operative ileus. Aliment Pharmacol Ther, 25:47-57.

Uemura K, Tatewaki M, Harris MB, et al. 2004. Magnitude of abdominal incision affects the duration of postoperative ileus in rats. Surg Endosc, 18:606-10.

Velez JP, Lince LF, Restrepo JI, et al. 1997. Early enteral nutrition in gastrointestinal surgery: a pilot study. Nutrition, 13:442-5.

Viscusi ER, Goldstein S, Witkowski T, et al. 2006. Alvimopan, a peripherally acting mu-opioid receptor antagonist, compared with placebo in postoperative ileus after major abdominal surgery: results of a randomized, double-blind, controlled study. Surg Endosc, 20:64-70.

Wolff BG, Michelassi F, Gerkin TM, et al. 2004. Alvimopan, a novel, peripherally acting mu opioid antagonist: results of a multicenter, randomized, double-blind, placebo-controlled, phase III trial of major abdominal surgery and postoperative ileus. Ann Surg, 240:728-35. 
